\title{
Fast Globally Optimal Segmentation of Cells in Fluorescence Microscopy Images
}

\author{
Jan-Philip Bergeest and Karl Rohr \\ University of Heidelberg, BIOQUANT, IPMB, and DKFZ Heidelberg \\ Dept. Bioinformatics and Functional Genomics, Biomedical Computer Vision Group
}

\begin{abstract}
Accurate and efficient segmentation of cells in fluorescence microscopy images is of central importance for the quantification of protein expression in high-throughput screening applications. We propose a new approach for segmenting cell nuclei which is based on active contours and convex energy functionals. Compared to previous work, our approach determines the global solution. Thus, the approach does not suffer from local minima and the segmentation result does not depend on the initialization. We also suggest a numeric approach for efficiently computing the solution. The performance of our approach has been evaluated using fluorescence microscopy images of different cell types. We have also performed a quantitative comparison with previous segmentation approaches.
\end{abstract}

\section{Introduction}

Cell nucleus segmentation is one of the most important tasks in analyzing and quantifying fluorescence microscopy images. In particular, in high-throughput applications, semi-automatic and manual analysis are not feasible because of the enormous amount of image data. Automatic methods are needed which efficiently deal with different cell types and image artifacts such as intensity inhomogeneities.

In recent years, different approaches for the segmentation of cell nuclei in fluorescence microscopy images have been introduced. Often thresholding approaches are applied which, however, suffer from intensity inhomogeneities within nuclei and over a whole image. To separate clustered nuclei, watershed-based techniques are frequently used (e.g. [137]). A main class of cell segmentation approaches is based on deformable models, which allow incorporation of a priori knowledge and can capture a wide spectrum of different shapes. One can distinguish between parametric models and implicit models (e.g. [1249148105]). Implicit representations based on level sets have gained increased interest since topological changes can be handled naturally. In most of these approaches regionbased energy functionals are employed. However, a disadvantage of previous level set approaches is that the underlying energy functionals lead to non-convex minimization problems. Hence, the optimization function has local minima and the global solution is generally not found (using local optimization methods which are typically applied). In addition, the segmentation result depends on the initialization.

G. Fichtinger, A. Martel, and T. Peters (Eds.): MICCAI 2011, Part I, LNCS 6891, pp. 645-652 2011.

(C) Springer-Verlag Berlin Heidelberg 2011 
In this contribution, we introduce a new approach for cell nucleus segmentation in fluorescence microscopy images. Compared to previous work, our approach is based on two energy functionals that lead to convex minimization problems for which global solutions are determined. Also, we take advantage of the combination of the region-based functional of Chan-Vese [2] and the Bayesian functional proposed in [1] which was used for cell segmentation in [5]. A convex formulation for the region-based functional in [2] was derived in [6] and we employ this scheme for splitting clustered cell nuclei. In addition, we use the Bayesian functional in [1] to cope with intensity inhomogeneities. We reformulate this functional leading to a convex optimization problem. For both functionals we propose an efficient minimization scheme based on the Split Bregman method. We have successfully applied our approach to two different sets of 2D fluorescence microscopy images comprising different cell types and we have compared the results with previous segmentation approaches.

\section{Cell Nuclei Segmentation}

Our approach for the segmentation of cell nuclei in fluorescence microscopy images is based on two energy functionals. In this section, we first describe the original non-convex formulation of the functionals. Then we introduce a reformulation as convex optimization problem and we suggest an efficient numerical technique for finding the solution.

\subsection{Non-convex Energy Functionals}

The first functional used in our approach is the region-based energy functional proposed by Chan-Vese [2]:

$$
E_{1}(\Theta, \partial \Omega)=\lambda\left(\kappa_{0} \int_{\Omega_{0}}\left(I(\mathbf{x})-\mu_{0}\right)^{2} d \mathbf{x}+\kappa_{1} \int_{\Omega_{1}}\left(I(\mathbf{x})-\mu_{1}\right)^{2} d \mathbf{x}\right)+\operatorname{Per}\left(\Omega_{1}\right),
$$

with $\Theta=\left(\mu_{0}, \mu_{1}\right)$, where $\mu_{0}$ and $\mu_{1}$ are the mean intensities of the background region $\Omega_{0}$ and the foreground region $\Omega_{1}$, respectively. $\partial \Omega$ denotes the boundaries between the regions $\Omega_{i}, I(\mathbf{x})$ are the image intensities at position $\mathbf{x}, \operatorname{Per}\left(\Omega_{1}\right)$ is the perimeter of $\Omega_{1}$, and $\kappa_{0}, \kappa_{1}, \lambda$ are weighting factors. As the second functional, we use the one proposed in [11, which was derived based on a Bayesian approach:

$$
E_{2}(\Theta, \partial \Omega)=\lambda\left(\int_{\Omega_{0}}-\log P\left(I(\mathbf{x}) \mid \Omega_{0}\right) d \mathbf{x}+\int_{\Omega_{1}}-\log P\left(I(\mathbf{x}) \mid \Omega_{1}\right) d \mathbf{x}\right)+\operatorname{Per}\left(\Omega_{1}\right),
$$

with $\Theta=\left(\mu_{0}, \mu_{1}, \sigma_{0}, \sigma_{1}\right)$, where $\mu_{i}$ and $\sigma_{i}$ are the mean intensities and standard deviations of the regions $\Omega_{i} . P\left(I(\mathbf{x}) \mid \Omega_{i}\right)$ is the conditional probability that pixel $\mathbf{x}$ with intensity $I(\mathbf{x})$ belongs to region $\Omega_{i}$. Here, we assume a Gaussian distribution $P\left(I(\mathbf{x}) \mid \Omega_{i}\right)=\frac{1}{\sqrt{2 \pi} \sigma_{i}} e^{-\frac{\left(I(\mathbf{x})-\mu_{i}\right)^{2}}{2 \sigma_{i}^{2}}}$. An advantage of $E_{2}$ compared to $E_{1}$ is that the weights $\kappa_{0}$ and $\kappa_{1}$ are not needed, but are implicitly included via 
the standard deviations $\sigma_{i}$, which are estimated from the image data. Using a level set representation for $E_{1}$ and $E_{2}$ and applying the Euler-Lagrange equation leads to the gradient flow for the level set function $\phi$

$$
\frac{\partial \phi(\mathbf{x})}{\partial t}=\left(-\lambda r_{j}+\nabla \cdot \frac{\nabla \phi}{|\nabla \phi|}\right) H^{\prime}(\phi(\mathbf{x})),
$$

with $H(x)^{\prime}$ being the derivative of the Heaviside function $H(x)=\{0$ if $x<$ $0,1$ if $x \geq 0\}$. The term $r_{j}$ corresponds to the external image forces of the two energies $E_{j}, j=1,2$, which are defined as

$$
\begin{aligned}
& r_{1}=\kappa_{1}\left(I(\mathbf{x})-\mu_{1}\right)^{2}-\kappa_{0}\left(I(\mathbf{x})-\mu_{0}\right)^{2} \\
& r_{2}=\log P\left(I(\mathbf{x}) \mid \Omega_{1}\right)-\log P\left(I(\mathbf{x}) \mid \Omega_{0}\right)
\end{aligned}
$$

The parameter vector $\Theta$ can be computed directly:

$$
\begin{aligned}
\mu_{0} & =\frac{\int I(\mathbf{x})(1-H(\phi(\mathbf{x}))) d \mathbf{x}}{\int(1-H(\phi(\mathbf{x}))) d \mathbf{x}} & \mu_{1} & =\frac{\int I(\mathbf{x}) H(\phi(\mathbf{x})) d \mathbf{x}}{\int H(\phi(\mathbf{x})) d \mathbf{x}} \\
\sigma_{0}^{2} & =\frac{\int\left(I(\mathbf{x})-\mu_{0}\right)^{2}(1-H(\phi(\mathbf{x}))) d \mathbf{x}}{\int(1-H(\phi(\mathbf{x}))) d \mathbf{x}} & \sigma_{1}^{2} & =\frac{\int\left(I(\mathbf{x})-\mu_{1}\right)^{2} H(\phi(\mathbf{x})) d \mathbf{x}}{\int H(\phi(\mathbf{x})) d \mathbf{x}}
\end{aligned}
$$

\subsection{Convex Energy Functionals}

In 1], it has been shown that certain non-convex minimization problems can be reformulated as convex problems. There, a convex formulation for $E_{1}$ in (1) was derived. In our approach, we use this formulation and we also derive a convex formulation for $E_{2}$ in (2). Following [1], the Heaviside function in (3) is omitted since (3) and the following gradient descent equation have the same steady state solution

$$
\frac{\partial \phi(\mathbf{x})}{\partial t}=-\lambda r_{j}+\nabla \cdot \frac{\nabla \phi}{|\nabla \phi|} .
$$

Then, the corresponding energy functional can be stated as

$$
E_{j}(\Theta, \phi)=\lambda\left\langle\phi, r_{j}\right\rangle+|\nabla \phi|_{1}
$$

where $\langle\cdot, \cdot\rangle$ denotes the inner product, $|\cdot|_{1}$ is the $L 1$-norm, and $|\nabla \phi|_{1}$ corresponds to $\operatorname{Per}\left(\Omega_{1}\right)$. By restricting $\phi$ to lie in a finite interval, e.g. $[0,1]$, the global minimum can be guaranteed by solving the following convex problem for the normalized $\phi_{n}$ :

$$
\min _{0 \leq \phi_{n} \leq 1} E_{j}\left(\Theta, \phi_{n}\right)=\lambda\left\langle\phi_{n}, r_{j}\right\rangle+\left|\nabla \phi_{n}\right|_{1}
$$

\subsection{Split Bregman Method}

To solve (8) we use the Split Bregman method. This method is a general technique for efficiently solving L1-regularized problems and for iteratively finding extrema of convex functionals [6]. The method consists of variable splitting 
and Bregman iteration. Variable splitting is achieved by introducing the auxiliary vector $\boldsymbol{d}$ (with dimension according to the image domain) and by using a quadratic penalty term to enforce the constraint $\boldsymbol{d}=\nabla \phi_{n}$. For (8) this leads to

$$
\left(\phi_{n}^{*}, \boldsymbol{d}^{*}\right)=\arg \min _{0 \leq \phi_{n} \leq 1, \boldsymbol{d}}\left(\lambda\left\langle\phi_{n}, r_{j}\right\rangle+|\boldsymbol{d}|_{1}+\frac{\nu}{2}\left|\boldsymbol{d}-\nabla \phi_{n}\right|_{2}^{2}\right),
$$

where $\phi_{n}^{*}$ and $\boldsymbol{d}^{*}$ denote the iteratively computed solution. In (9) $\nabla \phi_{n}$ is no longer associated with the $L 1$-norm, however, the constraint $\boldsymbol{d}=\nabla \phi_{n}$ is only weakly enforced. To enforce the constraint exactly, the Bregman iteration technique is applied. With this technique a vector $\boldsymbol{b}$ is included in the penalty function and an alternating minimization is carried out:

$$
\begin{aligned}
\left(\phi_{n}^{k}, \boldsymbol{d}^{k}\right) & =\arg \min _{0 \leq \phi_{n} \leq 1, \boldsymbol{d}}\left(\lambda\left\langle\phi_{n}, r_{j}\right\rangle+|\boldsymbol{d}|_{1}+\frac{\nu}{2}\left|\boldsymbol{d}-\nabla \phi_{n}-\boldsymbol{b}^{k-1}\right|_{2}^{2}\right), \\
\boldsymbol{b}^{k} & =\boldsymbol{b}^{k-1}+\nabla \phi_{n}^{k}-\boldsymbol{d}^{k},
\end{aligned}
$$

where $\phi_{n}^{k}$ and $\boldsymbol{d}^{k}$ represent the solution at iteration $k$. The problem in (10), (11) is first solved w.r.t. $\phi_{n}$, while $\boldsymbol{d}$ and $\boldsymbol{b}$ are fixed and second w.r.t. $\boldsymbol{d}$. In our case, we use a fast iterative Gauss-Seidel solver for the first minimization. The second minimization is obtained explicitly using the vector-valued shrinkage operator:

$$
\boldsymbol{d}^{k}=\max \left\{\left|\boldsymbol{b}^{k-1}+\nabla \phi_{n}^{k}\right|_{2}-\nu, 0\right\} \frac{\boldsymbol{b}^{k-1}+\nabla \phi_{n}^{k}}{\left|\boldsymbol{b}^{k-1}+\nabla \phi_{n}^{k}\right|_{2}}
$$

\subsection{Globally Optimal Cell Segmentation}

Our approach for cell nuclei segmentation combines the two convex energy functionals $E_{1}$ and $E_{2}$ in (8). The functional $E_{2}$ is used to deal with intensity inhomogeneities over the whole image and to segment cell nuclei with varying intensities. $E_{1}$ is used to split cell nuclei which have been falsely merged using $E_{2}$ and is minimized independently for all regions that were segmented using $E_{2}$. Note, that doing this we use multiple level sets for the segmentation of an image. This is similar to multiple level set approaches (e.g. 124914 8]). However, in our case we do not need a coupling term to prevent level sets from merging because we perform the minimization within previously segmented regions.

Our approach consists of three steps. In the first step, we perform a segmentation of the whole image using the functional $E_{2}$. Since this functional comprises parameters for both the mean intensities and the variances of objects we can deal with varying background intensities and with cell nuclei with varying intensities. However, cell nuclei that are close to each other are likely to be merged in this step (see Fig. 1a ). In the second step, we minimize $E_{1}$ for each of the segmented cell nuclei regions in the first step. This allows splitting of clustered cell nuclei (see Fig. 1b). In the third step, we use $E_{2}$ again but this time for the segmented objects from the second step within a region of interest around the object. The third step is needed because the second step cannot deal with inhomogeneities within cell nuclei. The result of the third step in Fig. 1c shows that the segmentation at the border of the nuclei is more accurate and smoother compared to 


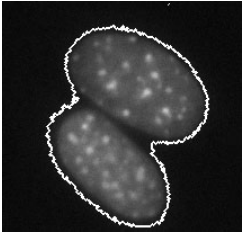

(a)

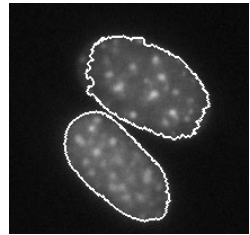

(b)

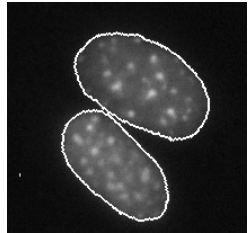

(c)

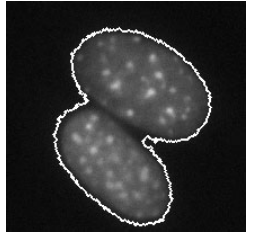

(d)

Fig. 1. Original images overlaid with contours of the segmentation results. Result after the first step (a) the second step (b) and the third step (c) (d) shows the result when using $E_{2}$ in the second step.

the result after the second step. Note, that if we would use $E_{2}$ in the second step then clustered nuclei could not be separated because the contrast in the region between the cells is not large enough (see Fig. 11d).

\section{$3 \quad$ Experimental Results}

We have applied our approach to two sets of $2 \mathrm{D}$ fluorescence microscopy images of cell nuclei of different cell types from 3 for which ground truth is available. The first data set consists of 48 images with a size of $1349 \times 1030$ pixels which include in total 1831 U20S Hoechst stained cell nuclei (see Fig. 2a ). The second data set contains 49 images with a size of $1344 \times 1024$ pixels comprising in total 2178 NIH3T3 Hoechst stained nuclei (see Fig. 2d). Note, that several images in the second data set are heavily affected by intensity inhomogeneities and visible artifacts. Therefore, automatic analysis of the second set is more challenging compared to the images in the first set.

To evaluate the performance of our approach we determined region-based and contour-based measures. As region-based measure we used the Dice coefficient and as contour-based measures we employed the normalized sum of distances (NSD) 3 and the Hausdorff distance. The Dice coefficient is defined as $\operatorname{Dice}(R, S)=\frac{2|R \cap S|}{|R|+|S|}$, where $R$ is the binary reference image, and $S$ is the binary segmented image. The NSD is defined as $\operatorname{NSD}(R, S)=\frac{\sum_{i \in R \cup S \backslash R \cap S} D(i)}{\sum_{i \in R \cup S} D(i)}$, where $D(i)$ is the minimal Euclidean distance of pixel $i$ to the contour of the reference object. The Hausdorff distance is defined as $h(R, S)=\max _{i \in S_{c}}\{D(i)\}$, with $S_{c}$ being the contour of the segmented object. We also used two detection measures, namely the number of false positives (FP) and the number of false negatives (FN). FP corresponds to spuriously segmented nuclei and FN corresponds to nuclei that have not been segmented. In all our experiments we used $\nu=10$ and $\kappa_{0}=1, \kappa_{1}=1$ in (10),(11). In the first and second step of our approach we used $\lambda=10000$ and in the third step we chose $\lambda=1000$.

Table 1 shows the results of our approach (after 1 step and after all 3 steps) for the different performance measures averaged over all images of each data set. As a comparison we also show the results for Otsu thresholding as well as the 


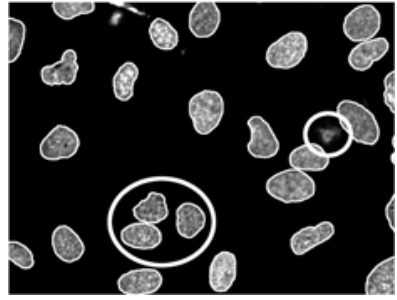

(a)

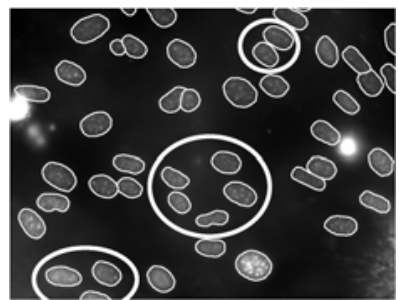

(d)

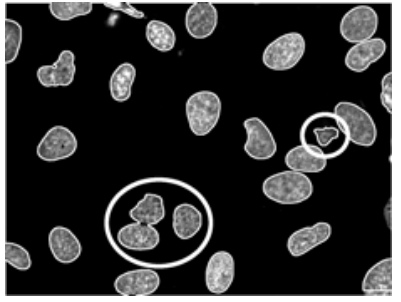

(b)

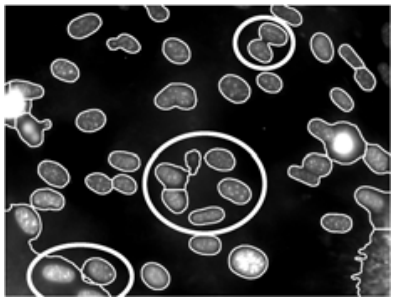

(e)

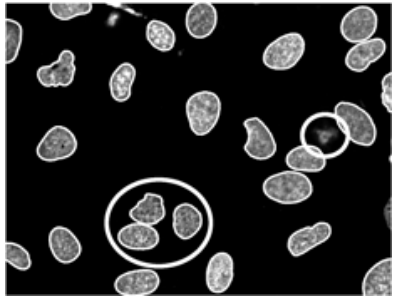

(c)

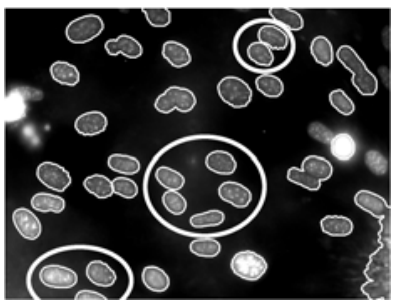

(f)

Fig. 2. Original images with overlaid contours of segmentation results. First row: U20S cells, second row: NIH3T3 cells; first column: ground truth, second column: merging algorithm [7], third column: our approach.

watershed algorithm and the region merging algorithm [7] applied to the mean thresholded image. The latter approach uses an improved watershed algorithm followed by statistical model-based region merging to cope with oversegmentation and yielded the best results in the study in 33. Also, we included the result of using the non-convex functional $E_{2}$ in (2). In addition, we included the result of manual segmentation (for a subset of 5 images for each data set) by a different observer than the one who provided the ground truth (second row, Manual). It can be seen that our approach yields the best result for the Dice coefficient and the NSD for both data sets, and that the result is comparable to the result by the different observer (Manual). In particular, for the more challenging NIH3T3 images we obtain significantly better results than previous approaches. Regarding the Hausdorff distance, our approach yields better results for the NIH3T3 cell images, while the results are equally good as those for the algorithm in [7] for the U20S images. Furthermore, it can be seen that our approach (3 steps) yields better results than our 1 step approach and thus the multiple step scheme is advantageous. However, the 1 step approach still yields better results than using the non-convex functional $E_{2}$ in (2). For the FP value the result is equally good or similar to the best results of the other approaches. Regarding the FN value we obtain for the U20S cell images similar results as for the other approaches, while for the NIH3T3 cell images we obtain intermediate results. However, note that in biological applications a small $\mathrm{FP}$ value is more important than a small FN value. The reason is that it is often necessary to quantify the signal intensity in a second channel within segmented nucleus regions, and FP regions 
Table 1. Quantitative results for the different approaches

\begin{tabular}{|c||c|c|c|c|c||c|c|c|c|c|}
\hline \multirow{2}{*}{ Approach } & \multicolumn{3}{c||}{ U20S cells (48 images) } & \multicolumn{4}{c|}{ NIH3T3 cells (49 images) } \\
\cline { 2 - 11 } & Dice & NSD & Hausdorff & FP & FN & Dice & NSD & Hausdorff & FP & FN \\
\hline \hline Manual & 0.93 & 0.04 & 9.8 & 0.6 & 2.2 & 0.87 & 0.07 & 12.1 & 0.0 & 3.2 \\
\hline Otsu & 0.87 & 0.12 & 34.8 & 0.3 & 5.5 & 0.64 & 0.35 & 36.7 & 1.7 & 26.4 \\
\hline Watershed & 0.69 & 0.36 & 34.3 & 1.9 & 3.0 & 0.62 & 0.37 & 19.1 & 11.6 & 5.5 \\
\hline Merging algorithm [7] & 0.92 & 0.08 & 13.3 & 1.0 & 3.3 & 0.70 & 0.28 & 19.0 & 7.0 & 5.8 \\
\hline Non-convex $E_{2}$ in (2) & 0.88 & 0.15 & 25.2 & 1.5 & 2.8 & 0.76 & 0.24 & 21.9 & 3.5 & 5.2 \\
\hline Our approach (1 step) & 0.93 & 0.11 & 18.1 & 1.4 & 3.2 & 0.84 & 0.23 & 21.0 & 3.3 & 8.6 \\
\hline Our approach (3 steps) & 0.94 & 0.06 & 13.3 & 0.5 & 3.9 & 0.83 & 0.14 & 16.5 & 1.7 & 11.3 \\
\hline
\end{tabular}

have a strong influence on the statistical analysis. Compared to the approach in [7] our approach allows better splitting of clustered cell nuclei and spurious objects are not included (see Fig. 2b]c), and our approach yields a more accurate segmentation of cell nuclei shapes (see Fig. 2eff).

In our approach, the minimization of $E_{2}$ in (8) on the whole image converges after about 5 iterations and the computation time is approximately 15 seconds per image. In comparison, using a standard level set scheme for the non-convex energy $E_{2}$ in (2) with gradient descent optimization needs about 400 iterations and the computation time is about 9 minutes. Thus, our approach is significantly faster. As convergence criterion we used the average Euclidean distance between the contours of segmented objects in two successive iterations.

\section{Discussion}

We have introduced a new approach based on active contours for cell nuclei segmentation in fluorescence microscopy images. Our approach employs two convex energy functionals for which globally optimal solutions are determined. By combining the two functionals we can cope with clustered cells, different cell types, and intensity inhomogeneities. To minimize the energy functionals we suggested using the Split Bregman method which significantly reduces the computation time compared to standard level set approaches. Thus, our approach is suitable for high-throughput applications and very large data sets. We have demonstrated the applicability of our approach using 97 real fluorescence microscopy images comprising in total 4009 cells of two different cell types. It turned out that our approach yields superior results compared to previous approaches.

Acknowledgments. We thank Luis Pedro Coelho (CMU) for help with his software. Support of the BMBF project FANCI (SysTec) is gratefully acknowledged. 


\section{References}

1. Chan, T.F., Esedoglu, S., Nikolova, M.: Algorithms for finding global minimizers of image segmentation and denoising models. SIAM J. Appl. Math. 66, 1632-1648 (2006)

2. Chan, T.F., Vese, L.A.: Active contours without edges. IEEE Trans. Image Process 10(2), 266-277 (2001)

3. Coelho, L.P., Shariff, A., Murphy, R.F.: Nuclear segmentation in microscope cell images: a hand-segmented dataset and comparison of algorithms. In: ISBI 2009, pp. 518-521 (2009)

4. Dufour, A., Shinin, V., Tajbakhsh, S., Guillen-Aghion, N., Olivo-Marin, J.C., Zimmer, C.: Segmenting and Tracking Fluorescent Cells in Dynamic 3-D Microscopy With Coupled Active Surfaces. IEEE Trans. Image Process 14(9), 1396-1410 (2005)

5. Dzyubachyk, O., van Cappellen, W.A., Essers, J., Niessen, W.J., Meijering, E.H.W.: Advanced Level-Set-Based Cell Tracking in Time-Lapse Fluorescence Microscopy. IEEE Trans. Med. Imag. 29(3), 852-867 (2010)

6. Goldstein, T., Bresson, X., Osher, S.: Geometric Applications of the Split Bregman Method: Segmentation and Surface Reconstruction. J. of Scientific Computing 45(1), 272-293 (2010)

7. Lin, G., Adiga, U., Olson, K., Guzowski, J.F., Barnes, C.A., Roysam, B.: A hybrid $3 \mathrm{D}$ watershed algorithm incorporating gradient cues and object models for automatic segmentation of nuclei in confocal image stacks. Cytometry A 56(1), 23-36 (2003)

8. Mosaliganti, K., Gelas, A., Gouaillard, A., Noche, R., Obholzer, N., Megason, S.: Detection of Spatially Correlated Objects in 3D Images Using Appearance Models and Coupled Active Contours. In: Yang, G.-Z., Hawkes, D., Rueckert, D., Noble, A., Taylor, C. (eds.) MICCAI 2009. LNCS, vol. 5762, pp. 641-648. Springer, Heidelberg (2009)

9. Nath, S.K., Palaniappan, K., Bunyak, F.: Cell segmentation using coupled level sets and graph-vertex coloring. In: Larsen, R., Nielsen, M., Sporring, J. (eds.) MICCAI 2006. LNCS, vol. 4190, pp. 101-108. Springer, Heidelberg (2006)

10. Padfield, D., Rittscher, J., Thomas, N., Roysam, B.: Spatio-temporal cell cycle phase analysis using level sets and fast marching methods. Med. Image Anal. 13(1), 143-155 (2009)

11. Rousson, M., Deriche, R.: A Variational Framework for Active and Adaptative Segmentation of Vector Valued Images. In: Proc. Workshop Motion Video Comput., pp. 56-62. IEEE Computer Soc., Los Alamitos (2002)

12. Ortiz de Solorzano, C., Malladi, R., Lelievre, S., Lockett, S.J.: Segmentation of nuclei and cells using membrane related protein markers. J. Micr. 201(3), 404-415 (2001)

13. Wählby, C., Lindblad, J., Vondrus, M., Bengtsson, E., Björkesten, L.: Algorithms for cytoplasm segmentation of fluorescence labelled cells. Analytical Cellular Pathology 24(2), 101-111 (2002)

14. Yan, P., Zhou, X., Shah, M., Wong, S.T.C.: Automatic segmentation of highthroughput RNAi fluorescent cellular images. IEEE Trans. Inf. Techn. in Biomed 12(1), 109-117 (2008) 